\title{
Approximation Hardness of Minimum Edge Dominating Set and Minimum Maximal Matching
}

\author{
Miroslav Chlebík ${ }^{1}$ and Janka Chlebíková ${ }^{*}$ \\ 1 Max Planck Institute for Mathematics in the Sciences \\ Inselstraße 22-26, D-04103 Leipzig, Germany \\ chlebik@mis.mpg.de \\ ${ }^{2}$ Christian-Albrechts-Universität zu Kiel \\ Institut für Informatik und Praktische Mathematik \\ Olshausenstraße 40, D-24098 Kiel, Germany \\ jch@informatik.uni-kiel.de
}

\begin{abstract}
We provide the first interesting explicit lower bounds on efficient approximability for two closely related optimization problems in graphs, Minimum Edge Dominating Set and Minimum Maximal MATChing. We show that it is NP-hard to approximate the solution of both problems to within any constant factor smaller than $\frac{7}{6}$. The result extends with negligible loss to bounded degree graphs and to everywhere dense graphs.
\end{abstract}

\section{Introduction}

We consider two NP-hard optimization problems Minimum Edge Dominating Set and Minimum Maximal Matching. The problems are motivated by their important applications in areas such as telephone switching networking.

An edge dominating set for a simple graph $G=(V, E)$ is a subset $D$ of $E$ such that for all $e \in E \backslash D$ there is an edge $f \in D$ such that $e$ and $f$ are adjacent. The Minimum Edge Dominating Set problem (Min-Eds) asks to find an edge dominating set of minimum cardinality, eds $(G)$ (resp. minimum total weight in weighted case). The decision version of MiN-EDS was shown by Yannakakis and Gavril to be NP-complete even on graphs which are planar (or bipartite) of maximum degree 3 [14]. Later Horton and Kilakos extended their results showing that NP-completeness holds also for planar bipartite graphs, line graphs, total graphs, perfect claw-free graphs, and planar cubic graphs [10]. On the other hand, the problem admits polynomial-time approximation scheme (PTAS) for planar graphs [1] or $\lambda$-precision unit disk graphs [11]. Some special classes of graphs for which the problem is polynomially solvable have been discovered, e.g. trees [12], claw-free chordal graphs, locally connected claw-free graphs, the

\footnotetext{
* The author has been supported by EU-Project ARACNE, Approximation and Randomized Algorithms in Communication Networks, HPRN-CT-1999-00112.
} 
line graphs of total graphs, the line graphs of chordal graphs [10], bipartite permutation graphs, cotriangulated graphs [13].

An edge dominating set of minimum cardinality has close relationship with minimum maximal matchings. A matching $M \subseteq E$ in a graph $G=(V, E)$ is maximal if no other matching in $G$ properly contains it (or, equivalently, if a matching is an edge dominating set). The Minimum Maximal Matching problem (Min-MaXL-Match) asks to find a maximal matching of minimum cardinality. The fact that in $G$ there are much more edge dominating sets than maximal matchings does not distinguish the optimization problems Min-EDS and Min-MaXl-Match significantly. In fact, the minimum cardinality of edge dominating sets in $G$ is achieved also on maximal matchings in $G$. Even more interestingly, there is a simple polynomial-time algorithm to transform any given edge dominating set $D$ in $G$ to a maximal matching $M$ in $G$ with size $|M| \leq|D|$ (see e.g. [14]). This fact makes Min-EdS and Min-MaxL-Match equivalent. Any polynomial time $\rho$-approximation algorithm for Min-EDS can be easily transformed to the one for Min-MAXL-MATCH with the same performance ratio; the converse relation being trivial. It is easy to observe that no matching in a graph $G$ can be more than twice larger than any maximal matching. Therefore constructing any maximal matching (which is possible in $O(|E|)$ time) suffices to approximate even the search version of Min-Eds and Min-MaXl-Match problems to within a factor of 2. Recently, also weighted Min-EDS was shown to be approximable efficiently to within 2 ([8]).

It is quite straightforward via simple reduction (see [2]) that weighted MiNEds is at least as hard to approximate as Minimum Node Cover (Min-NC), hence any inapproximability result for Min-NC applies directly to weighted Min-EDS. In particular, the result of Dinur and Safra [7] implies that it is NPhard to approximate weighted MiN-EDS to within any constant factor smaller than $10 \sqrt{5}-21 \approx 1.36067$.

In (unweighted) Min-EDS, or equivalently, Min-MaXL-Match, the gap between the upper bound 2 and the known lower bound on approximability, is much wider. The transformation of Yannakakis and Gavril ([14]) showing NPcompleteness of Min-MAXL-MaTCH reducing 3-Min-NC (the restriction of Min$\mathrm{NC}$ to cubic graphs) to it, may be regarded as an $L$-reduction and hence gives APX-completeness for problems we are interested in. This implies NP-hardness to approximate Min-MAXL-MatCH to within a factor $1+\delta$ for some $\delta>0$. But lower estimates on $\delta$ obtained from inapproximability results for 3-Min-NC, and from parameters of that $L$-reductions are only about $\frac{1}{500}$.

In Section 2 we prove that it is NP-hard to approximate the problem MiNEDS (and hence also Min-MAXL-MATch) to within any factor smaller than $\frac{7}{6}$. We present two approaches how to achieve this lower bound. The first relates the problem to parameters in PCP characterization of NP class, the second one capitalizes on inapproximability result for linear equations systems. The lower bound $\frac{7}{6}-\delta$ holds also for graphs with maximum degree $B$ (reffered to as $B$-instances in the table), where the value $B$ depends on $\delta$. We have slightly better lower bounds for sparse bipartite graphs with all nodes but one of degree 
$B$ (reffered to as $B^{*}$-instances in the table); namely we prove NP-hardness factors of $1+\frac{1}{390}$ and $1+\frac{1}{250}$ for $B=4$ and $B=5$ respectively. The following table summarizes results from this contribution. The upper bound for all problems is 2 , except Maximum Total Matching with upper bound of $\frac{3}{2}$.

\begin{tabular}{|l|c|}
\hline Problem & Lower bound \\
\hline \hline Min-Eds or Min-MaxL-MATCH & $\frac{7}{6}-\delta$ \\
\hline B-Min-Eds or B-Min-MAXL-MATCH & $\frac{7}{6}-\frac{24 \log B}{B}$ \\
\hline Min-Eds or Min-MAXL-MATCH & $\frac{7+\theta}{6+2 \theta}-\delta$ \\
on everywhere $\theta$-dense graphs & \\
\hline 3 -Min-Eds & $1+\frac{1}{487}$ \\
\hline $4^{*}$-Min-Eds & $1+\frac{1}{390}$ \\
\hline $5^{*}$-Min-Eds & $1+\frac{1}{250}$ \\
\hline Maximum Total Matching & $\frac{10}{9}-\delta$ \\
\hline
\end{tabular}

Definitions. In a graph $G=(V, E)$ a set $C \subseteq V$ is a node cover, if every $e \in E$ is incident to some node in $C$. The Minimum Node Cover problem asks to find a node cover of minimum cardinality, $n c(G)$. A matching in a graph $G$ is a set of edges with no shared endpoints. A matching in $G$ is perfect if each node of $G$ is incident to an edge of this matching. For a constant $\theta \in(0,1)$, everywhere $\theta$-dense graph is a graph $G=(V, E)$ of minimum degree at least $\theta|V|$.

\section{General, bounded and dense instances}

Combinatorial analysis. It is easy to see that a set of edges $F \subseteq E$ is an edge dominating set of $G=(V, E)$ if and only if $V(F)$, the set of end nodes of edges in $F$, is a node cover of $G$. In particular, any maximal matching $M$ of $G$ (which is also an edge dominating set) satisfies

$$
2|M|=|V(M)| \geq n c(G) .
$$

Consequently, $n c(G) \leq 2 e d s(G)$ holds for every graph $G$. Those graphs $G$, for which the theoretical bound $n c(G)=2 e d s(G)$ is achieved, will be of our main interest in what follows.

Let us denote by $\mathcal{G}$ the class of graphs $G=(V, E)$ for which a minimum cardinality node cover $C \subseteq V$ of $G$ exists such that the subgraph induced by $C$ has a perfect matching. Clearly, any perfect matching $M^{\prime}$ in that subgraph is a maximal matching of $G$ (as its node set $V\left(M^{\prime}\right)=C$ is a node cover of $G)$. Moreover, as $2\left|M^{\prime}\right|=\left|V\left(M^{\prime}\right)\right|=|C|=n c(G), M^{\prime}$ is a minimum maximal matching of $G$ (due to $(1)$ ). Hence, we have just verified that $n c(G)=2 e d s(G)$ for every $G \in \mathcal{G}$. 
Due to this simple relation between $\operatorname{eds}(G)$ and $n c(G)$ in the class $\mathcal{G}$, our goal is to prove suitable NP-hard gap results for MiN-NC problem restricted to $\mathcal{G}$. For this purpose we have to show first that $\mathcal{G}$ is rich enough. In fact, we will deal with even more restricted class $\mathcal{G}_{0} \subseteq \mathcal{G}$ of graphs $G=(V, E)$ for which every minimal (on inclusion) node cover $C \subseteq V$ of $G$ induces a subgraph with a perfect matching.

We start with some combinatorial notions:

Definition 1. A graph $G[s]=(V[s], E[s])$ is an s-padding (s being a positive integer) of a graph $G=(V, E)$, if $G[s]$ is obtained from $G$ by replacing every node $v \in V$ by a set $v[s]$ of distinct nodes, $v[s]=\left\{v_{1}, v_{2}, \ldots, v_{s}\right\}$ and $E[s]:=$ $\left\{\left\{u_{i}, v_{j}\right\}:\{u, v\} \in E, i, j \in\{1,2, \ldots, s\}\right\}$.

This graph operation has been frequently used and many of its basic properties are well known. Clearly, whenever $C \subseteq V$ is a node cover of $G$, then $C[s]:=\cup_{v \in C} v[s]$ is a node cover of $G[s]$. Moreover, every minimal (on inclusion) node cover of $G[s]$ is of the form $C[s]$ for some (necessarily minimal) node cover $C$ of $G$. In particular, $n c(G[s])=s \cdot n c(G)$.

A graph whose $s$-padding has a perfect matching will be called $s$-matchable. Notice that any graph that admits covering of its node set by (pairwise) node disjoint subgraphs that are either copies of $K_{2}$ or (odd) cycles, is 2-matchable. A (nonempty) graph $G=(V, E)$ is said to be $s$-safe in what follows if for every node cover $C$ of $G$ the subgraph induced by $C$ is $s$-matchable. Clearly, this makes sense only for even positive integer $s$; for $s$ odd either $V$, or $V$ without any node, is a node cover with odd number of nodes, and it cannot induce $s$-matchable subgraph. Example of 2-safe graphs trivially are cliques $K_{r}$ with $r \geq 3$, and $K_{r}$ without any edge with $r \geq 5$ as well.

Theorem 1. Let $s$ be an even positive integer, and $G=(V, E)$ be a graph with the following property: there is a partition $V_{1} \cup V_{2} \cup \cdots \cup V_{p}$ of the node set $V$ such that for each $i \in\{1,2, \ldots, p\}$ the induced subgraph $G_{i}=\left(V_{i}, E_{i}\right)$ of $G$ is $s$-safe. Then the s-padding of $G$, the graph $G[s]$, has the following property: every minimal node cover of $G[s]$ induces a subgraph in $G[s]$ with a perfect matching. Hence $G[s] \in \mathcal{G}_{0} \subseteq \mathcal{G}$, and eds $(G[s])=\frac{1}{2} n c(G[s])=\frac{s}{2} n c(G)$.

The special case of Theorem 1 , when $s=2$ and all those graphs $G_{i}(i=$ $1,2, \ldots, p)$ are cliques of size at least 3 , is enough to consider to prove the main Theorem 3 of this paper. Such graphs naturally appear in a general reduction (so called FGLSS-reduction) from languages having efficient PCP (Probabilistic Checking of Proof) systems to approximation versions of MAXIMUM INDEPENDent Set (or Maximum Clique) and Minimum Node Cover.

\section{PCP based proof}

We will show that the problem Min-EDs relates in a straightforward way to parameters of PCP systems. We assume that the reader is familiar with the standard PCP terminology. Recall some notation for verifiers and the parametric 
complexity classes: Verifier $V$ is called $(r, q)$-restricted if on input $x$ it generates a random string $R$ tossing $r(|x|)$ coins and queries to an alleged membership proof $\pi$ via oracle access $q(|x|)$ times. Then it outputs $V^{\pi}(x, R) \in\{$ accept $=1$, reject $=0\}$.

A language $L$ belongs to the class $\mathrm{PCP}_{c, s}[r, q]$, where $c, s$ are completeness and soundness probabilities, if there exists an $(r, q)$-restricted verifier $V$ that given an input $x$ and oracle access to $\pi$ has the following properties: for $x \in L$ there is a membership proof $\pi$ such that the verifier accepts $\pi$ with probability $\geq c$ (over all random strings $R \in\{0,1\}^{r(|x|)}$ ); for $x \notin L$ and each membership proof $\pi$ the probability that the verifier $V$ accepts $\pi$ is $<s$.

For a verifier $V$ and an input $x$ the graph $G_{x}$ (more precisely $G_{V, x}$ ), the FGLSS graph corresponding to $V$ and $x$, is defined as follows: Every node in $G_{x}$ corresponds to an accepting configuration $(R, Q) \in\{0,1\}^{r(|x|)} \times\{0,1\}^{q}$ of $V$ 's computation. That means, for each random string $R$ we enumerate the $2^{q}$ possible binary sequences that represent possible sequence of answers to $V$ 's oracle queries. For each such sequence $Q$, we include the pair $(R, Q)$ as a node of $G_{x}$ if $V$ accepts the sequence $Q$ on random string $R$. The edges of $G_{x}$ correspond to inconsistencies among these configurations. That is, there is an edge between $(R, Q)$ and $\left(R^{\prime}, Q^{\prime}\right)$ if there is a query $\pi[i]$ that will be asked by $V$ on both $(x, R)$ and $\left(x, R^{\prime}\right)$, and it has different responses in $Q$ and $Q^{\prime}$.

The accepting configurations of the form $(R, \cdot)$ for a fixed random string $R$ form a layer. Each layer clearly induces a clique in $G_{x}$. A verifier has average free bit complexity $f_{a v}:=f_{a v}(|x|)$ if the sum of sizes of layers is $2^{r(|x|)+f_{a v}(|x|)}$. Notice, that this is the number of nodes of the graph $G_{x}$.

For application to problems like MiN-NC it is important that $f_{a v}$ is bounded above by small constant, $f_{*}$, independent of $|x|$. For our application to MinEDS it is further important that we can work with verifiers for which all layers have size at least 3 . Then clearly 2-padding of $G_{x}$ satisfies, due to Theorem 1, $e d s\left(G_{x}[2]\right)=n c\left(G_{x}\right)$.

An independent set in $G_{x}$ corresponds to a proof for $x$ and the size of this set is $2^{r}$ times the probability that $V$ accepts this proof. Thus if $x \in L$ there is an independent set of size $c 2^{r}$ (hence $n c\left(G_{x}\right) \leq 2^{r}\left(2^{f_{a v}}-c\right)$ ), whereas if $x \notin L$ the size of any independent set in $G_{x}$ is less than $s 2^{r}$ (and hence $n c\left(G_{x}\right)>2^{r}\left(2^{f_{a v}}-\right.$ $s)$ ). As $\frac{2^{f a v}-s}{2^{f a v}-c}=1+\frac{c-s}{2^{f a v}-c} \geq 1+\frac{c-s}{2^{f}-c}$, any algorithm that approximates eds (on graphs $G_{x}[2]$ ) to within $1+\frac{c-s}{2^{f_{*}-c}}$ would be sufficient to decide if $x \in L$.

The reduction above has polynomial time complexity if $r(x)=O(\log |x|)$ and $q$ is a constant. Hence if for some NP-complete language $L$ there is a proof that $L \in \mathrm{PCP}_{c, s}[O(\log |x|), q]$ using verifier $V$ with average free bit complexity $\leq f_{*}$ ( $f_{*}$ being constant) and with at least 3 accepting configurations for any random string $R$, then approximation of eds to within $1+\frac{c-s}{2^{f_{*}-c}}$ is NP-hard. Applying Håstad's result [9] that for every $\varepsilon \in\left(0, \frac{1}{4}\right) \mathrm{NP} \subseteq \mathrm{PCP}_{1-\varepsilon, 0.5+\varepsilon}[O(\log x), 3]$ using verifier with $q=3$ queries, and exactly 4 accepting configurations for any random string $R$ (hence $f_{a v}=f_{*}=2$ ), we obtain inapproximability of MIN-EDS to within any constant smaller than $\frac{7}{6}$. 


\section{Reduction from linear equation systems}

Definition 2. MAX-E3-LIN-2 is the following optimization problem: Given a system I of linear equations over $\mathbb{Z}_{2}$, with exactly 3 (distinct) variables in each equation. The goal is to maximize, over all assignments $\varphi$ to the variables, the ratio $\frac{\text { sat }(\varphi)}{I \mid}$, where sat $(\varphi)$ is the number of equations of I satisfied by $\varphi$. Ek-MAX-E3-LIN-2 denote the same maximization problem, where each variable occurs exactly $k$ times.

Let $Q(\varepsilon, k)$ be the following partial decision subproblem of MAX-E3-Lin-2: for given instance of Ek-MAX-E3-LIN-2 the problem is to decide if the fraction of more than $(1-\varepsilon)$ or less than $\left(\frac{1}{2}+\varepsilon\right)$ of all equations is satisfied by the optimal (i.e. maximizing) assignment. The following result follows from Håstad results $[9]$ and the proof can be found in [4]

Theorem 2. For every $\varepsilon \in\left(0, \frac{1}{4}\right)$ there is a constant $k(\varepsilon)$ such that for every $k \geq k(\varepsilon)$ the partial decision subproblem $Q(\varepsilon, k)$ of MAX-E3-Lin-2 is NP-hard.

Notation. Denote $F(x):=-x \log x-(1-x) \log (1-x), x \in(0,1)$, where $\log$ means the natural logarithm. Further, $G(c, t):=(F(t)+F(c t)) /(F(t)-$ $\left.\operatorname{ct} F\left(\frac{1}{c}\right)\right)$ for $0<t<\frac{1}{c}<1, g(t):=G\left(\frac{1-t}{t}, t\right)$ for $t \in\left(0, \frac{1}{2}\right)$. More explicitly, $g(t)=2[-t \log t-(1-t) \log (1-t)] /[-2(1-t) \log (1-t)+(1-2 t) \log (1-2 t)]$. Using Taylor series of the logarithm near 1 we see that the denominator here is $t^{2} \cdot \sum_{k=0}^{\infty} \frac{2^{k+2}-2}{(k+1)(k+2)} t^{k}>t^{2}$, and $-(1-t) \log (1-t)=t-t^{2} \sum_{k=0}^{\infty} \frac{1}{(k+1)(k+2)} t^{k}<t$, consequently $g(t)<\frac{2}{t}\left(1+\log \frac{1}{t}\right)$.

For large enough $B$ we look for $\delta \in\left(0, \frac{1}{6}\right)$ such that $6\left\lfloor g\left(\frac{\delta}{2}\right)\right\rfloor+12 \leq B$. As $g\left(\frac{1}{12}\right) \approx 75.62$ and $g$ is decreasing in $\left(0, \frac{1}{12}\right)$, we can see that for $B \geq 462$ any $\delta>\delta(B):=2 g^{-1}\left(\left\lfloor\frac{B}{6}\right\rfloor-1\right)$ will do. Trivial estimates on $\delta(B)$ (using $g(t)<$ $\left.\frac{2}{t}\left(1+\log \frac{1}{t}\right)\right)$ are $\delta(B)<\frac{24}{B-12}(\log (B-12)+1-\log 12)<\frac{24 \log B}{B}$.

We will need the following lemma (based on Theorem 6.6 in [3]) about regular bipartite expanders to prove the main Theorem 3.

Lemma 1. Let $t \in\left(0, \frac{1}{2}\right)$ and $d$ be an integer for which $d>g(t)$. For every sufficiently large positive integer $n$ there is a d-regular $n$ by $n$ bipartite graph $H$ with bipartition $\left(V_{0}, V_{1}\right)$, such that for each independent set $J$ in $H$ either $\left|J \cap V_{0}\right| \leq t n$, or $\left|J \cap V_{1}\right| \leq t n$.

Theorem 3. For every $\delta \in\left(0, \frac{1}{6}\right)$ it is NP-hard to approximate Min-Eds (MinMAXL-MATCH) to within $\frac{7}{6}-\delta$, even in graphs of degree at most $6\left\lfloor g\left(\frac{\delta}{2}\right)\right\rfloor+12 \leq$ $6\left\lceil\frac{4}{\delta}\left(1+\log \frac{2}{\delta}\right)\right\rceil+6$. Consequently, for any $B \geq 462$ it is NP-hard to approximate $B$-Min-EdS ( $B$-Min-MaXL-Match) to within any constant smaller than $\frac{7}{6}-$ $\delta(B)$, where $\delta(B):=2 g^{-1}\left(\left\lfloor\frac{B}{6}\right\rfloor-1\right)<\frac{24}{B-12}(\log (B-12)+1-\log 12)<\frac{24 \log B}{B}$. Further, for any $\theta \in(0,1)$, it is NP-hard to approximate Min-EDs (Min-MAXL$\mathrm{MATCH}$ ) on everywhere $\theta$-dense graphs to within any constant smaller than $\frac{7+\theta}{6+2 \theta}$. 
Sketch of the proof. (a) We first prove the result for graphs without restriction on degrees. Fix $\delta \in\left(0, \frac{1}{6}\right)$, choose $\varepsilon \in\left(0, \frac{1}{4}\right)$ such that $\frac{7}{6}-\delta<\frac{7-2 \varepsilon}{6+2 \varepsilon}$, and then $k$ for which $Q(\varepsilon, k)$ is NP-hard. We describe simple reduction $f$ from E $k$-MAX-E3-LIN-2 to graphs and check how the NP-hard gap of $Q(\varepsilon, k)$ is preserved for the value of $e d s$ or $n c$.

Let $I$ be an instance of E $k$-MAX-E3-Lin-2, $\mathcal{V}(I)$ be the set of variables of $I$, and $m:=|\mathcal{V}(I)|$. Clearly the system $I$ has $\frac{m k}{3}$ equations. For each equation we take simple gadget, a 4-clique. More precisely, if the equation reads as $x+y+z=j(j \in\{0,1\})$ we take a 4 -clique whose nodes has labels $x y z=00 j$, $x y z=01(1-j), x y z=10(1-j)$ and $x y z=11 j$. Notice, that nodes correspond to partial assignments to variables making the equation satisfied. Now we add an edge for each pair of inconsistently labeled nodes. The pair of nodes is inconsistent if a variable $u \in \mathcal{V}(I)$ exists that is assigned differently in their labels. Let us denote the graph we obtained by $G_{I}, f(I):=G_{I}[2]$ its 2-padding.

Clearly $G_{I}$ has $\frac{4}{3} m k$ nodes. By Theorem $1, G_{I}[2] \in \mathcal{G}$, hence $\operatorname{eds}\left(G_{I}[2]\right)=$ $\frac{1}{2} n c\left(G_{I}[2]\right)=n c\left(G_{I}\right)$. Denote by $\alpha\left(G_{I}\right)$ cardinality of the maximum independent set in $G_{I}$. We show that $\alpha\left(G_{I}\right)=\frac{m k}{3} \cdot \operatorname{OPT}(I)$, where $\operatorname{OPT}(I)$ is the fraction of maximum cardinality of satisfiable equations over all assignment.

Given any assignment $\varphi: \mathcal{V}(I) \rightarrow\{0,1\}$, let $J_{\varphi}$ consists of all nodes whose partial assignment is the restriction of $\varphi . J_{\varphi}$ is an independent set and $\left|J_{\varphi}\right|$ is just the number of equations from $I$ that are satisfied by $\varphi$. Hence $\left|J_{\varphi}\right| \leq \frac{m k}{3} \mathrm{OPT}(I)$ for each assignment, and there is an assignment for which the equality holds. Moreover, for any independent set $J$ in $G_{I}$ there is an assignment $\varphi$ such that $J \subseteq J_{\varphi}$. Hence the union of those partial assignments is the restriction of some assignment $\varphi: \mathcal{V}(I) \rightarrow\{0,1\}$. Now $\alpha\left(G_{I}\right)=\frac{m k}{3} \mathrm{OPT}(I)$ easily follows. Further, $n c\left(G_{I}\right)=\frac{4}{3} m k-\alpha\left(G_{I}\right)=\frac{m k}{3}(4-\mathrm{OPT}(I))$. Hence the NP-hard question of whether $\mathrm{OPT}(I)$ is greater than $(1-\varepsilon)$, or in the interval $\left\langle\frac{1}{2}, \frac{1}{2}+\varepsilon\right)$ is transformed to NP-hard partial decision problem of whether $n c\left(G_{I}\right)=e d s\left(G_{I}[2]\right)$ is less than $\frac{m k}{3}(3+\varepsilon)$, or it is in the interval $\left(\frac{m k}{3}\left(\frac{7}{2}-\varepsilon\right), \frac{m k}{3} \cdot \frac{7}{2}\right\rangle$.

(b) To prove inapproximability within $\frac{7}{6}-\delta$ for bounded degree graphs one can use the idea already used in [6]: to replace graph $G_{I}$ of all inconsistencies by its lower degree subgraph with suitable expanding properties.

Let $\delta \in\left(0, \frac{1}{6}\right)$ be given, put $d:=\left\lfloor g\left(\frac{\delta}{2}\right)\right\rfloor+1\left(\leq\left\lceil\frac{4}{\delta}\left(1+\log \frac{2}{\delta}\right)\right\rceil\right)$. Then we choose $t \in\left(0, \frac{\delta}{2}\right)$, close enough to $\frac{\delta}{2}$, so that $d>g(t)$. Further we choose $\varepsilon \in\left(0, \frac{1}{4}\right)$ such that $\left(\frac{7}{2}-\varepsilon-6 t\right) /(3+\varepsilon)>\frac{7}{6}-\delta$. Then a positive integer $k$ is chosen so that (i) $Q(\varepsilon, k)$ is NP-hard (see Theorem 2), and (ii) there is a $d$-regular $2 k$ by $2 k$ bipartite graph $H$ with bipartition $\left(V_{0}, V_{1}\right)$, such that for each independent set $J$ in $H$ either $\left|J \cap V_{0}\right| \leq 2 k t$, or $\left|J \cap V_{1}\right| \leq 2 k t$ (see Lemma 1). Keep one such $H$ fixed from now on.

Now we start with an instance $I$ of E $k$-MAX-E3-Lin-2, with $m:=|\mathcal{V}(I)|$. We take the same equation gadget as in part (a). Consider a variable $u \in \mathcal{V}(I)$. Let $V_{j}(u)(j \in\{0,1\})$ be the set of all $2 k$ nodes in which $u$ has assigned bit $j$. Now we create a graph $G_{I}^{H}$ on the same set of nodes as $G_{I}$ (from the part (a)) but with maximum degree at most $3 d+3$, as follows: For each $u \in \mathcal{V}(I)$ we take edges between $V_{0}(u)$ and $V_{1}(u)$ exactly as prescribed by our fixed expander $H$. 
Having this done, one after another, for each $u \in \mathcal{V}(I)$, we get the graph $G_{I}^{H}$. Let $h(I):=G_{I}^{H}[2]$ be its 2-padding.

Clearly, the transformation $h$ is polynomial, $G_{I}^{H}$ is of degree at most $3 d+3$, and $h(I)$ is of degree at most $6 d+6$. Again, by Theorem $1, G_{I}^{H}[2] \in \mathcal{G}$, hence $\operatorname{eds}\left(G_{I}^{H}[2]\right)=\frac{1}{2} n c\left(G_{I}^{H}[2]\right)=n c\left(G_{I}^{H}\right)$. Clearly, any independent set in $G_{I}$ is also an independent set in $G_{I}^{H}$, hence $\alpha\left(G_{I}^{H}\right) \geq \alpha\left(G_{I}\right)=\frac{m k}{3} \mathrm{OPT}(I)$ and $n c\left(G_{I}^{H}\right) \leq$ $n c\left(G_{I}\right)=\frac{m k}{3}(4-\mathrm{OPT}(I))$.

On the other hand, we can show that $\alpha\left(G_{I}^{H}\right) \leq \alpha\left(G_{I}\right)+2 k m t=\frac{m k}{3}(\mathrm{OPT}(I)+$ $6 t)$ and $n c\left(G_{I}^{H}\right) \geq \frac{m k}{3}(4-\mathrm{OPT}(I)-6 t)$. Hence NP-hard question of whether OPT $(I)$ is greater than $(1-\varepsilon)$, or less than $\frac{1}{2}+\varepsilon$, is transformed to NP-hard partial decision problem of whether eds $\left(G_{I}^{H}[2]\right)=n c\left(G_{I}^{H}\right)$ is less than $\frac{m k}{3}(3+\varepsilon)$, or greater than $\frac{m k}{3}\left(\frac{7}{2}-\varepsilon-6 t\right)$.

(c) Let $\theta \in(0,1)$ be fixed and $r \in\left(1, \frac{7+\theta}{6+2 \theta}\right)$. To prove inapproximability to within $r$ on everywhere $\theta$-dense graphs, we choose $\varepsilon>0$ and $\omega>\frac{\theta}{1-\theta}$ such that $(7-2 \varepsilon+8 \omega) /(6+2 \varepsilon+8 \omega)>r$.

Now, as in part (a) starting from an instance $I$ create graph $G_{I}$. Consider the graph $G_{I}^{\prime}$ obtained from $G_{I}$ by adding a clique with $\left\lfloor\frac{4}{3} m k \omega\right\rfloor$ nodes and connecting any node of the clique to any node of $G_{I}$. It is easy to check that $G_{I}^{\prime}$ and $G_{I}^{\prime}[2]$ are everywhere $\theta$-dense (assuming $m k$ is large enough). By Theorem 1 , $G_{I}^{\prime}[2] \in \mathcal{G}$, hence eds $\left(G_{I}^{\prime}[2]\right)=n c\left(G_{I}^{\prime}\right)$. Moreover, $n c\left(G_{I}^{\prime}\right)=n c\left(G_{I}\right)+\left\lfloor\frac{4}{3} m k \omega\right\rfloor=$ $\frac{m k}{3}(4-\mathrm{OPT}(I))+\left\lfloor\frac{4}{3} m k \omega\right\rfloor$. Hence, OPT $(I)>1-\varepsilon$ implies eds $\left(G_{I}^{\prime}[2]\right)<\frac{m k}{6}(6+$ $2 \varepsilon+8 \omega)$, and $\operatorname{OPT}(I)<\frac{1}{2}+\varepsilon$ implies eds $\left(G_{I}^{\prime}[2]\right)>\frac{m k}{6}(7-2 \varepsilon+8 \omega)-1$.

\section{Sparse and small degree instances}

One of reductions of [14] starts with a cubic graph $G$ with $n$ nodes and produces a graph $f(G)$ with $10 n$ nodes and $\frac{21 n}{2}$ edges that is of maximal degree 3 and for which eds $(f(G))=2 n+n c(G)$. Using our (currently the best) inapproximability results for Min-NC problem on cubic graphs ([5]) one easily finds that it is NPhard to distinguish the case of $\operatorname{eds}(f(G))$ being larger than $2.51549586 n$ from that of being smaller than $2.5103305 n$. Hence inapproximability to within $1+\frac{1}{487}$ follows, even on instances produced by $f$.

Slightly better results can be obtained for sparse graphs for which one node is allowed to be of large degree and all the others have small degree. The following simple transformation $g$ from MiN-NC problem is universal. Given a graph $G=$ $(V, E)$ with $n$ nodes and $m$ edges, add one new special node 0 , connect 0 with every $u \in V$ by an edge, and replace every $e=\{u, v\} \in E$ by a simple gadget $G_{e}$ depicted on the following figure:

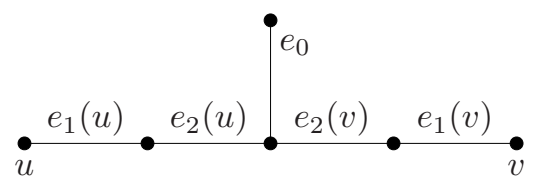


The bipartite graph $g(G)$ constructed in this way has $(n+4 m+1)$ nodes and $n+5 m$ edges. The important fact is that $\operatorname{eds}(g(G))$ is easily related to $n c(G)$. It can be proved that eds $(g(G))=m+n c(G)$.

Applying the reduction above to a cubic graph $G$ with $n$ nodes produces the bipartite graph $g(G)$ with $7 n+1$ nodes, $\frac{17}{2} n$ edges, and all nodes but one of degree $\leq 4$. On those instances the corresponding NP-hard question is to decide of whether eds $(g(G))$ is larger than 2.01549586n, or smaller than 2.0103305n, hence to approximate eds on such instances to within $1+\frac{1}{390}$ is NP-hard.

The results are slightly better starting with 4-regular graphs and using our NP-hard gap results [5] for them. For generic 4-regular graph $G$ with $n$ nodes the bipartite graph $g(G)$ has $9 n+1$ nodes, $11 n$ edges, and all nodes but one of degree $\leq 5$. Now it is NP-hard to decide of whether eds $(g(G))$ is larger than $2.53036437246 n$, or smaller than $2.52024291497 n$, hence to approximate eds on such instances to within $1+\frac{1}{250}$ is NP-hard.

Remarks. 1. Min-Eds is equivalent to the Minimum (Node) Dominating SET problem (Min-DS) restricted to line graphs. Hence this restricted version of Min-DS is APX-complete, has simple 2-approximation algorithm, but it is NP-hard to approximate to within $\frac{7}{6}-\delta$ for any $\delta>0$. Let us mention that for general graphs MiN-DS is not in APX; it is as hard to approximate as the set cover problem.

2. Recall that if $G=(V, E)$ then the total graph of $G$, denoted by $T(G)$, is defined as $T(G)=\left(V \cup E, E \cup E^{\prime} \cup E^{\prime \prime}\right)$, where $E^{\prime}=\{(e, v): e \in E, v \in V$ and $v$ is incident with $e\}$, and $E^{\prime \prime}=\{(e, f): e, f \in E$ are adjacent edges $\}$. One can prove that $\alpha(T(G))=|V(G)|-e d s(G)$ (see e.g. [14]). In the proof of Theorem 3 we produced instances $G=(V, E)$ with $n:=\frac{8}{3} m k$ nodes for which it was NPhard to distinguish between the case of $\operatorname{eds}(G)<\frac{n}{16}(6+2 \varepsilon)$ and the one of $e d s(G)>\frac{n}{16}(7-2 \varepsilon)$. For the problem MAX-IS in total graphs they translate as $\alpha(T(G))>\frac{n}{16}(10-2 \varepsilon)$ and $\alpha(T(G))<\frac{n}{16}(9+2 \varepsilon)$, respectively. Hence it is NPhard to approximate Maximum IndePEndent Set (MAX-IS) in total graphs (MaXimum Total Matching problem for $G$ ) to within any constant smaller than $\frac{10}{9}$. On the other hand, it is easy to design $\frac{3}{2}$-approximation algorithm for MAX-IS in $T(G)$, assuming the graph $G=(V, E)$ is given as an input. It suffices to find any maximal matching $M$ of $G$ and return $M \cup(V \backslash V(M))$; it is an independent set in $T(G)$ of size at least $\frac{2}{3} \alpha(T(G))$.

3. Passing to the complementary problem Min-NC in Remark 2 one gets $n c(T(G))=|E(G)|+e d s(G)$. To obtain an interesting explicit lower bound on approximability of MiN-NC in total graphs, one can use our NP-hard gap result for Min-EDS in sparse graphs. For example, NP-hard gap of $5 *$-Min-EdS transforms to the one showing that to approximate MiN-NC in total graphs within $1+\frac{1}{1336}$ is NP-hard. The NP-hard gap with the same inapproximability applies to Min-Eds (Min-MaXl-Match) in total graphs as well. This is due to the fact that in $T(G)$ any node cover with even number of nodes induces the graph with a perfect matching, assuming that $G$ was connected (see e.g. [10]). It implies that for a connected graph $G, \operatorname{eds}(T(G))=\left\lceil\frac{n c(T(G))}{2}\right\rceil$. 
We can go even further. Having NP-hard gap result for Min-EDs in total graphs, we can use Remark 2 for the graph $T(G)$ in place of $G$ to show the NP-hard gap result for MAX-IS of 2-iterated total graph of $G, T(T(G))$.

Using mathematical induction, for any positive integer $r$ we can derive explicit NP-hard gap result for each of problems MAX-IS, Min-NC, Min-EDS, Min-MAXL-MATCH restricted to the $r$-iterated total graphs.

The fact, that the lower bounds for polynomial time approximability of these problems converge very rapidly to 1 with increasing $r$, does not necessarily mean that those results are weak. In fact, one can show the upper bounds of the form $1+\delta^{r^{2}}$, for some constant $\delta \in(0,1)$, for these problems on the $r$-iterated total graphs.

\section{References}

1. B. S. Baker: Approximation algorithms for NP-complete problems on planar graphs, Journal of ACM 41(1994), 153-180.

2. R. Carr, T. Fujito, G. Konjevod and O. Parekh: A $2 \frac{1}{10}$-approximation algorithm for a generalization of the weighted edge-dominating set problem, Journal of Combinatorial Optimization 5(2001), 317-326.

3. F. R. K. Chung: Spectral Graph Theory, CBMS Regional Conference Series in Mathematics, American Mathematical Society, 1997, ISBN 0-8218-0315-8.

4. M. Chlebík and J. Chlebíková: Approximation hardness for small occurrence instances of NP-hard problems, Proc. of the 5th CIAC, LNCS 2653, 2003, Springer, 152-164 (also ECCC Report TR02-73, 2002).

5. M. Chlebík and J. Chlebíková: Inapproximability results for bounded variants of optimization problems, Proc. of the 14th Inter. Symp. on Fundamentals of Computation Theory (FCT), Malmö, Sweden, August 12-15, 2003, LNCS 2751, 2003, Springer (also ECCC Report TR03-26, 2003).

6. A. Clementi and L. Trevisan: Improved non-approximability results for vertex cover with density constraints, Theor. Computer Science 225(1999), 113-128.

7. I. Dinur and S. Safra: The importance of being biased, ECCC Report TR02-104, 2001.

8. T. Fujito and H. Nagamochi: A 2-approximation algorithm for the minimum weight edge dominating set problem, Discrete Appl. Math. 118(2002), 199-207.

9. J. Håstad: Some optimal inapproximability results, Journal of ACM 48(2001), 798-859.

10. J. D. Horton and K. Kilakos: Minimum edge dominating sets, SIAM J. Discrete Math. 6(1993), 375-387.

11. H. B. Hunt III, M. V. Marathe, V. Radhakrishnan, S. S. Ravi, D. J. Rosenkrantz and R. E. Stearns: A unified approach to approximation schemes for NP- and PSPACE-hard problems for geometric graphs, Proc. of the 2nd ESA, LNCS $\mathbf{8 5 5}$, 1994, 424-435.

12. S. Mitchell and S. Hedetniemi: Edge domination in trees, Proc. of the 8th Southearn Conf. on Combinatorics, Graph Theory, and Computing, 1977, 489-509.

13. A. Srinivasan, K. Madhukar, P. Nagavamsi, C. Pandu Rangan and M.-S. Chang: Edge domination on bipartite permutation graphs and cotriangulated graphs, Inf. Proc. Letters 56(1995), 165-171.

14. M. Yannakakis and F. Gavril: Edge dominating sets in graphs, SIAM J. Appl. Math. 38(1980), 364-372. 